\title{
Respiratory Tract Infections in Inflammatory Bowel Disease Patients Taking Vedolizumab: A Systematic Review and Meta-Analysis of Randomized Controlled Trials
}

\author{
Irene Marafini ${ }^{1 \dagger}$, Edoardo Troncone ${ }^{1 \dagger}$, Irene Rocchetti ${ }^{2}$ and Giovanni Monteleone ${ }^{1 *}$ \\ ${ }^{1}$ Chair of Gastroenterology, Department of Systems Medicine, University of Rome Tor Vergata, Rome, Italy, ${ }^{2}$ Statistical Office, \\ Superior Council of Judiciary, Rome, Italy
}

OPEN ACCESS

Edited by:

Jean Paul Deslypere, Esculape CRO (Belgium), Belgium

Reviewed by:

Fernando Gomollón,

University of Zaragoza, Spain Luca Antonioli,

University of Pisa, Italy

Brian Godman,

University of Strathclyde,

United Kingdom

*Correspondence:

Giovanni Monteleone

gi.monteleone@med.uniroma2.it

${ }^{+}$These authors have contributed equally to this work

Specialty section: This article was submitted to Pharmaceutical Medicine and

Outcomes Research,

a section of the journal

Frontiers in Pharmacology

Received: 14 August 2020 Accepted: 03 December 2020 Published: 22 January 2021

Citation:

Marafini I, Troncone E, Rocchetti I and Monteleone G (2021) Respiratory Tract Infections in Inflammatory Bowel Disease Patients Taking Vedolizumab: A Systematic Review and MetaAnalysis of Randomized Controlled Trials.

Front. Pharmacol. 11:585732. doi: 10.3389/fphar.2020.585732
The ongoing COVID-19 pandemic has raised concerns about the risk of SARS-CoV-2 infection in patients with Crohn's disease (CD) and patients with ulcerative colitis (UC) taking immunosuppressants or biologics. We conducted a systematic review and metaanalysis to assess the risk of respiratory infections in patients with inflammatory bowel disease (IBD) treated with vedolizumab. We searched PubMed, EMBASE and Scopus to identify randomized controlled trials (RCT) comparing vedolizumab to placebo in patients with IBD. Outcomes were the rate of respiratory tract infections (RTI), upper respiratory tract infections (URTI) and lower respiratory tract infections (LRTI) among patients receiving vedolizumab as compared with placebo. Pooled rates were reported as Odds Ratios (OR) with 95\% Confidence Interval (Cl). Eight RCT involving 3,287 patients (1873 CD and 1415 UC) were analyzed; 2,493 patients received vedolizumab and 794 received placebo. The rates of RTI and URTI were statistically higher in vedolizumab-treated patients compared to placebo [OR = 1.63; 95\% Cl (1.07-2.49); OR = 1.64 95\% Cl (1.07-2.53) respectively]. UC patients, but not CD patients, receiving vedolizumab had a higher risk to develop RTI and URTI [OR $=1.98 ; 95 \% \mathrm{Cl}(1.41-2.77) ; \mathrm{OR}=2.02 ; 95 \% \mathrm{Cl}(1.42-2.87)]$ compared to placebo-treated patients. The number of LRTI was small in both treatment groups. Data confirm the good safety profile of vedolizumab even though RTI were more frequent in patients receiving vedolizumab and the risk of URTIs was significantly higher in patients with UC.

Keywords: crohn' disease, ulcerative colitis, coronavirus, pneumonia, integrin, $\alpha 4 \beta 7$

\section{INTRODUCTION}

Vedolizumab is a humanized monoclonal antibody approved for the treatment of active Crohn's Disease (CD) and ulcerative Colitis (UC). Vedolizumab binds to the $\alpha 4 \beta 7$ integrin thus preventing interaction of such an integrin with the adhesion molecule mucosal vascular addressin cell adhesion molecule 1 (MAdCAM-1) expressed on the vascular endothelium. This determines inhibition of the trafficking of activated T and B lymphocytes to the gut (Soler et al., 2009; Marafini et al., 2014; Zundler et al., 2019). Phase-3, double-blind, placebo-controlled studies of vedolizumab in UC (GEMINI 1) and CD (GEMINI 2) showed that the drug is effective as induction and maintenance 
therapy for the treatment of both disorders (Feagan et al., 2013; Sandborn et al., 2013). Effectiveness of the drug was confirmed by many real-world observational studies (Chaparro et al., 2018; Christensen et al., 2018; Lenti et al., 2018; Plevris et al., 2019; Scarozza et al., 2020). It was also reported that patients with less severe disease and naïve to tumor necrosis factor (TNF) blockers are more likely to respond to vedolizumab (Dulai et al., 2016; Amiot et al., 2017; Favale et al., 2019; Hupe et al., 2020; Meserve and Dulai, 2020). Overall, the above studies showed also the drug is well-tolerated with an acceptable safety profile (Colombel et al., 2017). In particular, when data from GEMINI 1, GEMINI 2, and a long-term safety study in UC and $\mathrm{CD}$ were pooled, it was shown that the incidence of upper respiratory tract infections (URTI) was numerically higher in patients receiving vedolizumab compared with those receiving placebo, although this difference was not statistically significant (Feagan et al., 2018). However, the GEMINI two study showed that, during the maintenance phase, the incidences of infections and of serious infections, including respiratory tract infections (RTI), were higher with vedolizumab than with placebo (Sandborn et al., 2013). Today, this latter finding could be particularly relevant because of the outbreak of coronavirus disease 2019 (COVID-19) caused by the novel severe acute respiratory syndrome coronavirus 2 (SARS-CoV-2) (Munster et al., 2020; Zhu et al., 2020). The clinical spectrum of SARS$\mathrm{CoV}-2$ ranges from asymptomatic or mild respiratory disease to pneumonia with respiratory distress syndrome, which can in some cases lead to a fatal outcome (Guan et al., 2020). Although, inflammatory bowel disease (IBD) patients seem to have no increased risk to be infected with SARS-CoV-2 (Bezzio et al., 2020; Monteleone and Ardizzone, 2020), physicians need evidences to decide whether to continue or hold specific treatments during this pandemic. To determine the risk of RTIs with vedolizumab in patients with IBD, we performed a systematic review and meta-analysis of randomized clinical trials (RCTs).

\section{METHODS}

The methods of our analysis and inclusion criteria were based on Preferred Reporting Items for Systematic Reviews and MetaAnalyses (PRISMA) recommendations (Shamseer et al., 2015). Our protocol was registered with PROSPERO on May 2020, after searching for ongoing systematic reviews (protocol number: CRD42020185760).

\section{Data Source and Search Strategy}

A comprehensive literature search was performed using PubMed, Embase and Scopus (from inception up to May 2020) to identify RCTs regarding the safety of vedolizumab in IBD. The electronic search was supplemented by manual screening of the studies. We identified studies using the following medical subject headings $(\mathrm{MeSH})$ and keywords including: "vedolizumab", "alpha4beta7", " $\alpha 4 \beta 7$ ", “anti-integrin” "and 'inflammatory bowel disease'. The Medline search strategy was ("vedolizumab" [Supplementary Concept] OR “vedolizumab” [All Fields]) OR alpha4beta7 [All
Fields] OR anti-integrin [All Fields] OR alpha4beta7 [All Fields] AND ("inflammatory bowel diseases" [MeSH Terms] OR ("inflammatory" [All Fields] AND "bowel" [All Fields] AND "diseases" [All Fields]) OR "inflammatory bowel diseases" [All Fields] OR ("inflammatory" [All Fields] AND "bowel" [All Fields] AND "disease" [All Fields]) OR "inflammatory bowel disease" [All Fields]).

\section{Outcome Assessment}

The primary outcome measure of interest was the rate of RTIs among patients receiving vedolizumab compared with placebo. Secondary outcomes included rate of upper respiratory tract infections (URTI) and lower respiratory tract infections (LRTI) and sub-analysis according to disease type (CD or UC).

\section{Inclusion and Exclusion Criteria}

As for inclusion criteria, we considered RCTs reporting the incidence of adverse events (AE). We included only article in English and conducted in adult patients with IBD. We determined the number of each AE by article. RCTs conducted in healthy subjects receiving vedolizumab were excluded from the analysis. RCTs without an arm receiving placebo were excluded from the final analysis. Therefore, we excluded from the analysis head-to-head studies comparing either vedolizumab with other active drugs or different routes of vedolizumab administration. Subgroup analysis were also performed separately in CD and UC.

\section{Selection Process}

Two authors independently searched each of the potential trials to determine whether they were eligible for inclusion. Uncertainties or disagreements were resolved with a consensus among the authors. Full reports were obtained for all titles meeting the inclusion criteria. Full reports were retrieved even in case of any uncertainty. Then, the review authors decided whether to include the retrieved studies by screening the full reports.

\section{Data Extraction}

Using standardized forms, two reviewers (IM and ET) extracted data independently. Discrepancies were resolved by discussion. An arbitrator (GM) resolved unresolved disagreements. The following data were extracted from each of the included studies: year of publication, study design, study population, patients characteristics (age, gender, IBD type), study duration (follow-up) and treatment duration, number of RTI [nasopharyngitis, URTI, influenza-like syndrome, lower respiratory tract infections (LRTI), and pneumonia for vedolizumab and placebo-treated patients]. In the final analysis, RTI were divided in two groups: URTI (including also nasopharyngitis and influenza-like syndrome) and LRTI (including also pneumonia).

We included in the vedolizumab group all the patients who received at least one dose of vedolizumab during the study period. Therefore, patients who completed the induction with vedolizumab and then were assigned to maintenance therapy with placebo were included in the vedolizumab group. The 


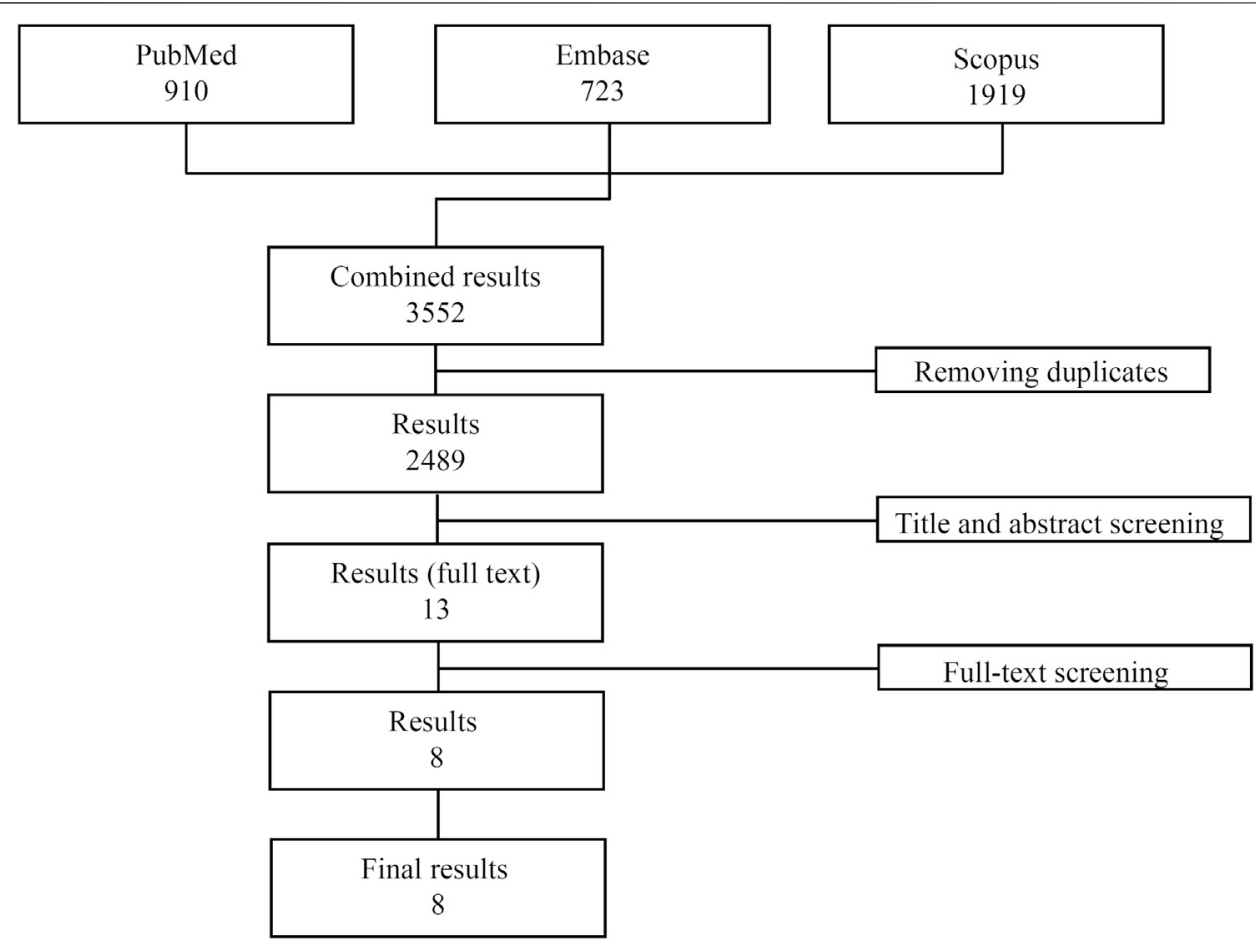

FIGURE 1 | Study flow-chart.

placebo group included patients who were never exposed to vedolizumab throughout the whole study duration.

\section{Statistical Analysis}

The global effect size was calculated taking into account the effects size of the single study analyses. Odds ratio (OR) was used to estimate total and single-study effect size. According to the existence of heterogeneity among the different studies, verified by applying the Q Cochran test (x2), random effect models (DerSimonian Laird method) were used to calculate pooled ratios for all the included studies and for the CD patients. The Mantel-Haenszel fixed effect model was used to calculate pooled OR for UC patients, given that the Q test in this case was not statistically significant. The statistical software $\mathrm{R}$ has been used for all the analysis made. Forests plots were provided to compare the OR of the single studies with respect to the combined Effect Size and to show graphically the confidence intervals and the significance of the estimates.

\section{RESULTS}

\section{Study Characteristics}

A total of 3,552 publications were initially identified (PubMed: 910, Embase: 723, Scopus: 1919). After removal of all the duplicates, 2,489 studies were evaluated and after evaluation of the abstracts, only 13 studies were considered for the full text revision. Of these, five studies were excluded: 1 because it was not a randomized controlled trial, 2 because the study population included healthy subjects only and 2 because they did not have a placebo group. Eight studies matching the inclusion/exclusion criteria were finally included in the analysis ((Feagan et al., 2013); (Sandborn et al., 2013); (Feagan et al., 2005; Feagan et al., 2008; Parikh et al., 2012; Sands et al., 2014; Motoya et al., 2019; Watanabe et al., 2020)) (Figure 1). All studies were multicentre, randomized placebo-controlled clinical trials published between 2005 and 2020 and conducted across Canada, United States, Europe, Russia and Japan. Overall, the mean treatment duration was 30.25 weeks (range 4-54 weeks), while the mean follow-up was 37.9 weeks (range 8-60 weeks). In total, 3,287 patients ( $1873 \mathrm{CD}$ and $1415 \mathrm{UC}$ ) were randomized: 2,493 exposed to at least one dose of vedolizumab and 794 receiving placebo. The mean age range across the studies was 35.7-43.8 for vedolizumab-treated patients and 32.6-44 for placebo-treated patients. One thousand-two hundred-eighty-six out of 2,493 vedolizumab-treated patients and 425 out of 794 placebo-treated patients were male.

\section{Respiratory Tract Infections are More Frequent in Patients Receiving Vedolizumab} Among vedolizumab-treated patients, 762 cases of RTI were reported: 731 were URTI and 31 were LRTI. Placebo-treated patients experienced 138 RTI: 133 URTI and 5 LRTI. URTI, including nasopharyngitis, were the most frequent RTI in both treatment groups (Table 1). The number of RTI was significantly higher in the group taking vedolizumab as compared to placebo $[\mathrm{OR}=1.63$; 95\% CI (1.07-2.49); Figure 2A]. When the analysis 
TABLE 1 | Number of patients enrolled in the indicated randomized, placebo-controlled clinical studies and number of respiratory tract infections in patients treated with either vedolizumab or placebo.

\begin{tabular}{|c|c|c|c|c|c|c|c|c|c|}
\hline References & $\begin{array}{c}\text { Patients } \\
\text { vedolizumab }\end{array}$ & $\begin{array}{l}\text { Patients } \\
\text { placebo }\end{array}$ & $\begin{array}{c}\text { Type } \\
\text { of IBD }\end{array}$ & RTI vedolizumab & $\begin{array}{c}\text { URTI } \\
\text { vedolizumab }\end{array}$ & $\begin{array}{c}\text { LRTI } \\
\text { vedolizumab }\end{array}$ & RTI placebo & $\begin{array}{c}\text { URTI } \\
\text { placebo }\end{array}$ & $\begin{array}{c}\text { LRTI } \\
\text { placebo }\end{array}$ \\
\hline Feagan et al. (2005) & 118 & 63 & UC & 16 & 16 & 0 & 5 & 5 & 0 \\
\hline Feagan et al. (2008) & 127 & 58 & $\mathrm{CD}$ & 19 & 19 & 0 & 3 & 3 & 0 \\
\hline Parikh et al. (2012) & 37 & 9 & UC & 8 & 8 & 0 & 4 & 4 & 0 \\
\hline Feagan et al. (2013) & 746 & 149 & UC & 224 & 193 & 31 & 27 & 22 & 5 \\
\hline Sandborn et al. (2013) & 967 & 148 & $C D$ & 381 & 381 & 0 & 62 & 62 & 0 \\
\hline Sands et al. (2014) & 209 & 207 & $\mathrm{CD}$ & 18 & 18 & 0 & 13 & 13 & 0 \\
\hline Motoya et al. (2019) & 210 & 82 & UC & 73 & 73 & 0 & 13 & 13 & 0 \\
\hline Watanabe et al. (2020) & 79 & 78 & $C D$ & 23 & 23 & 0 & 11 & 11 & 0 \\
\hline
\end{tabular}

IBD, inflammatory bowel disease; CD, Crohn's disease; UC, ulcerative colitis; RTI, respiratory tract infections; URTI, upper respiratory tract infections; LRTI, lower respiratory tract infections.

was restricted to URTI, the positive association between vedolizumab and RTI was confirmed [OR $=1.6495 \% \mathrm{CI}$ (1.07-2.53); Figure 2B). The number or LRTI was small in both treatment groups, and despite LRTI were numerically higher in patients taking vedolizumab compared to placebo (31 vs 5), no statistical analysis could be made.

Next, evaluations were performed separately for CD and UC. In CD, the risk of RTI and URTI was not statistically different between vedolizumab and placebo-treated patients [OR $=1.2$; 95\% CI (0.91-1.6) and OR $=1.2$; 95\% CI (0.91-1.6) respectively; Figure 3A,B]. In UC group, patients treated with vedolizumab had a higher risk to develop RTI or URTI than those receiving placebo $[\mathrm{OR}=1.98 ; 95 \% \mathrm{CI}(1.41-2.77) ; \mathrm{OR}=2.02 ; 95 \% \mathrm{CI}$ (1.42-2.87), respectively; Figure 4A,B].

\section{DISCUSSION}

The SARS-CoV-2 infection, which appeared in China in December 2019 and rapidly spread throughout the world, has forced clinicians to carefully evaluate the risk that IBD patients might have to develop COVID-19, as some drugs used to halt the IBD-associated tissue damaging inflammatory response may have an impact on infectious rates (Downey, 2016) and are known to increase incidence of RTI, including pneumonia (Kennedy et al., 2020; Sarzi-Puttini et al., 2020). At the same time, IBD by itself carries on an increased risk of pneumonia. We believe it is relevant to make a distinction between URTI and LRTI, as URTI are generally self-limiting diseases, while the latter are more difficult to treat and usually require the use of antibiotics (Godman et al., 2020).

In a retrospective cohort study, Long and colleagues demonstrated that the risk of developing pneumonia was approximately $50 \%$ higher for IBD patients than for the general population (Long et al., 2013). In a United States hospitalization database, Ananthakrishnan and colleagues showed that more than one fourth of all-related hospitalisations were attributable to infections, which caused excess mortality risk. Patients with pneumonia had the highest excess mortality risks compared with patients without infectionrelated hospitalization (Ananthakrishnan and McGinley, 2013). In these studies, the risk of infection among IBD patients was further enhanced by anti-TNF agents. Consistently, data emerging from a large prospective registry, indicate that the rates of serious infection rates, including RTI, were reported to be higher in the group of infliximab-treated CD patients than in patients receiving other non-biological therapies (Lichtenstein et al., 2018). In this context, it is however noteworthy that the risk of infection in patients receiving TNF blockers could be somewhat influenced by the underlying disease. Indeed, no increased risk of RTI was found in psoriatic patients treated with anti-TNF (Syed et al., 2020a). The risk of RTI following treatment with other biologics in psoriasis still has to be determined (Syed et al., 2020b).

The present meta-analysis was undertaken to determine whether patients exposed to vedolizumab have increased risk of RTI. Through analysis of the safety profile of vedolizumab documented in placebo-controlled RCTs involving 2,493 patients treated with vedolizumab and 794 treated with placebo, we showed that the number of RTI was significantly higher in the group of vedolizumab-treated patients than in the placebo group and this was evident for the URTI but not LRTI. Surprisingly, the risk of URTI among vedolizumab treated-patients was increased in UC but not in CD. Factors accounting for such a discrepancy remain unknown but it is conceivable that differences in the presence of confounders (e.g. smoking, concomitant use of steroids, immunesuppressors, or TNF blockers) among the two IBD populations can account for the increased risk of URTI in the vedolizumab-treated UC patients. However, we did not have access to the individual data of the patients enrolled in the RCTs and, therefore, we cannot ascertain the impact of such factors in the greater frequency of URTI in vedolizumab-treated UC patients.

The increased frequency of URTI in vedolizumab-treated UC patients might be explained by the expression of MAdCAM-1 in the oropharynx (Prados et al., 2018) and, therefore, vedolizumab treatment could block migration of $\mathrm{T}$ cells involved in the host defense against pathogens (e.g., $\mathrm{CD}^{+} \mathrm{T}$ cells) toward the upper respiratory mucosa.

Our results are conflicting with those published by Feagan et al., who demonstrated that in the two GEMINI RCTs $(n=1731$ patients) there was no statistical difference in terms of URTI between patients receiving vedolizumab and those receiving placebo (Feagan et al., 2018). However, in the same analysis, 
A

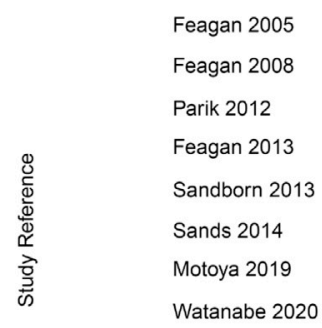

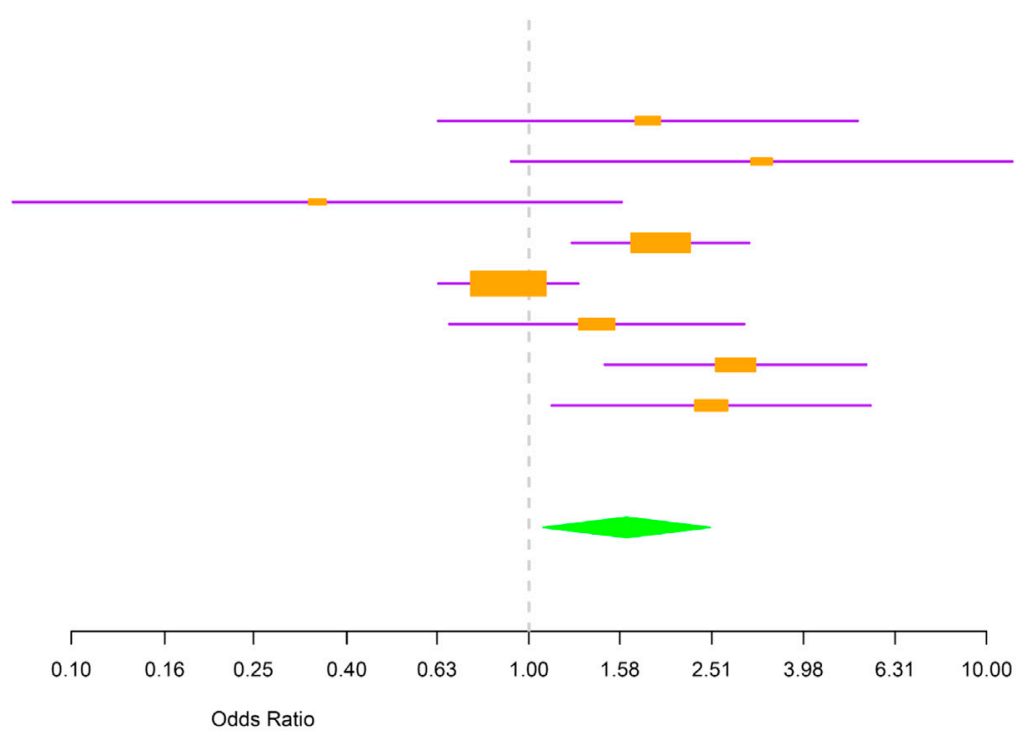

B

\begin{tabular}{|c|c|}
\hline & Feagan 2005 \\
\hline \multirow{7}{*}{ 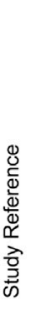 } & Feagan 2008 \\
\hline & Parik 2012 \\
\hline & Feagan 2013 \\
\hline & Sandborn 2013 \\
\hline & Sands 2014 \\
\hline & Motoya 2019 \\
\hline & Watanabe 2020 \\
\hline
\end{tabular}

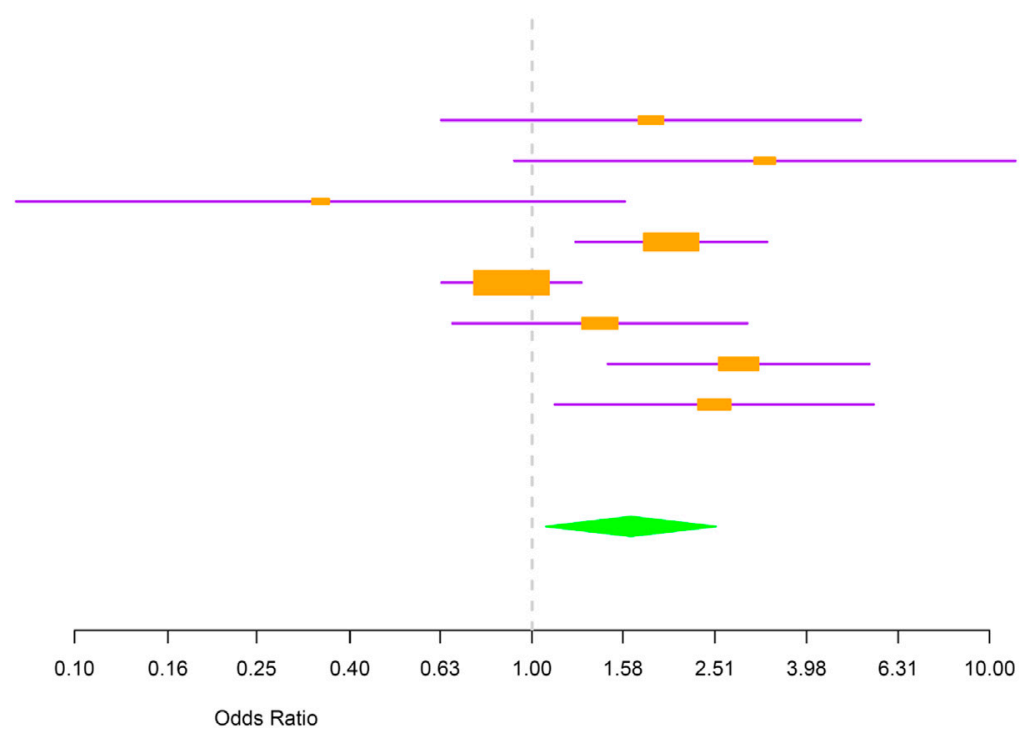

FIGURE 2 | (A) Forest plot for the rate of respiratory tract infections in inflammatory bowel disease patients. (B) Forest plot for the rate of upper respiratory tract infections in inflammatory bowel disease patients.

the authors reported that the incidence of URTIs was numerically higher in patients receiving vedolizumab compared with those receiving placebo. A possibility is that such a discrepancy reflects differences in the number of RCTs and patients analyzed. In fact, in contrast to the Feagan's analysis, we extended our evaluation to eight RCTs. Another possibility is that in the GEMINI studies, safety outcomes were evaluated separately for induction and maintenance therapy and for patients receiving vedolizumab at different intervals, while in our analysis all the vedolizumabtreated patients were grouped together. Moreover, in the assessment of $\mathrm{AE}$, both GEMINI studies included in the placebo group patients receiving vedolizumab in the induction phase and then assigned to placebo in the maintenance phase. In contrast, these patients were included in the vedolizumab group in our study as we cannot exclude the possibility that adverse events documented during the maintenance phase could depend on a carry-over effect of the drug. Consistently, our placebo group included exclusively those patients who never received vedolizumab treatment. Our data differ also, in part, from those published by Colombel and colleagues, who documented no increased risk of infections, including RTI, in patients exposed to vedolizumab. Notably, such an analysis included six doubleblind or open-label trials and did not take into consideration other RCTs evaluating the safety profile of vedolizumab, which were included in the present meta-analysis (Colombel et al., 2017).

In our study, the incidence of LRTI was low in both treatment groups, even though a trend toward higher rates was observed in 


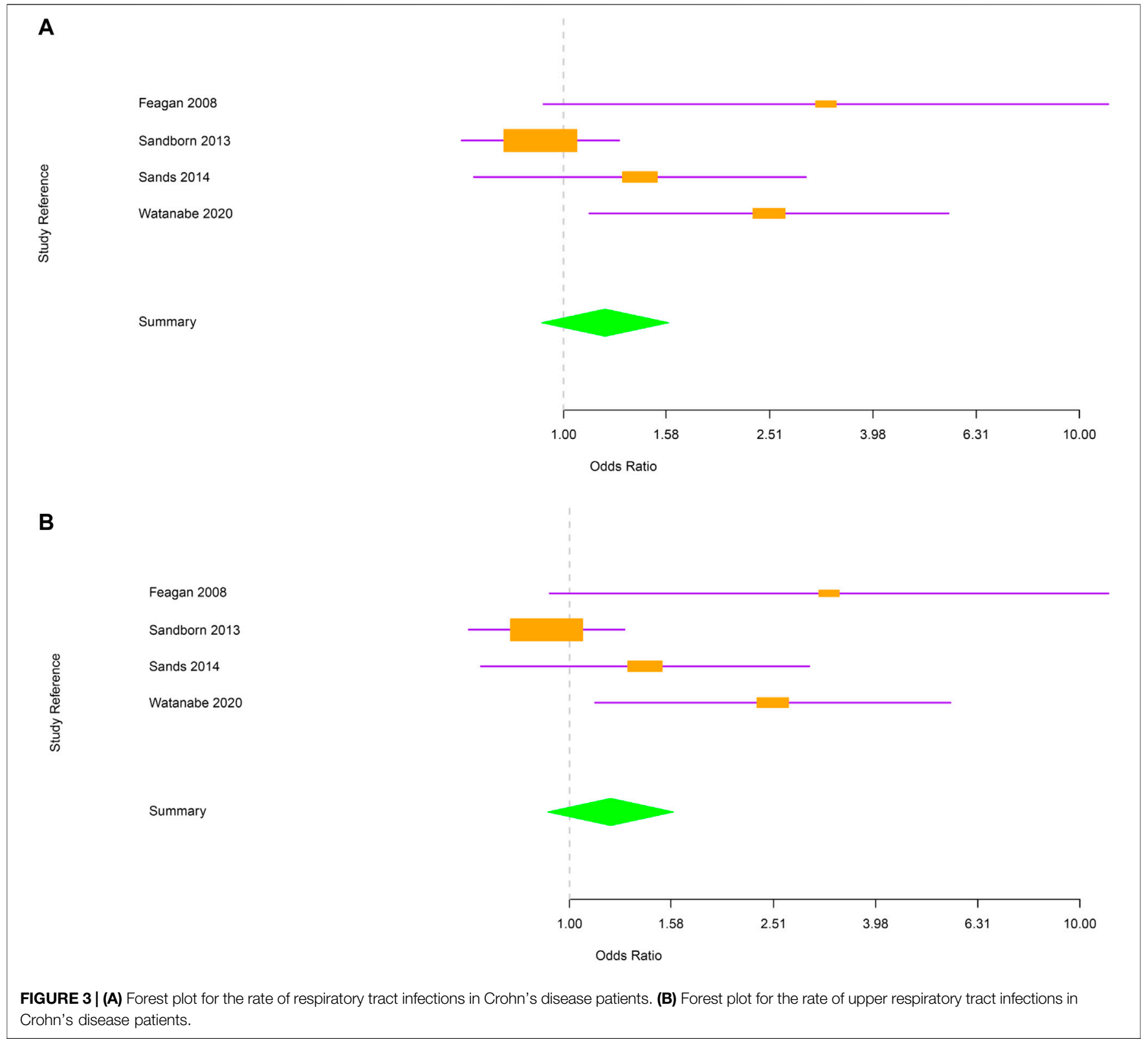

vedolizumab-treated patients. Interestingly, no pneumonia was evident from our study, while Feagan and colleagues reported that 11 vedolizumab-treated and two placebo-treated patients developed pneumonia, a finding that was not reported in the original GEMINI trial (Feagan et al., 2013; Sandborn et al., 2013). This reflects the fact that some RCTs indicate only AE occurring in more than $2-5 \%$ of the patients and some cases of bronchopneumonia can be classified as "any serious infection".

Comforting results on the use of other biologics are present in the latest literature. For example, in a large cohort study, adult COVID-19 patients with recent anti-TNF exposure did not have increased rates of hospitalization or mortality compared with patients without recent anti-TNF exposure (Yousaf et al., 2020).

We are aware that our study has some limitations. In RCTs, the screening procedure excludes the patients with potential risk factors for RTI (e.g,. cardio-vascular and respiratory comorbidities, older age). Thus, the carefully selected IBD patient population in RCTs does not reflect exactly the more refractory or complicated IBD patient populations treated in clinical practice. Moreover, the safety data considered in our analysis referred to studies with short-term follow-up, so we could not assess the long-term effect of vedolizumab on RTIs because the RCTs in our study had placebo-controlled periods of no more than 54 weeks. As mentioned above, additional confounders for RTI could not be evaluated due to the lack of individual patient data, even though in RCTs researchers randomize usually the patients taking into account all the known confounders.

We excluded non-controlled, real-world experiences from our analysis as they may not take into consideration minor adverse 


\section{A}

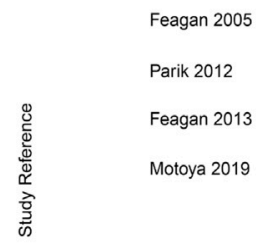

Summary

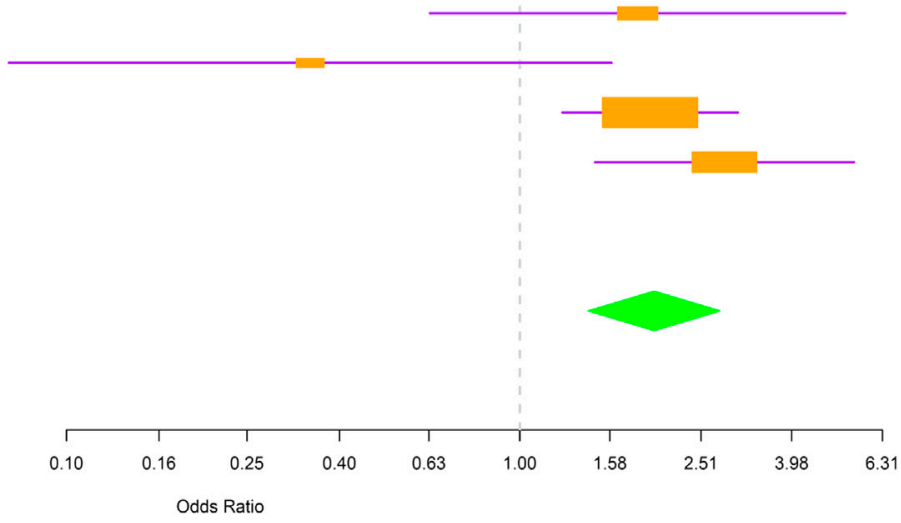

B

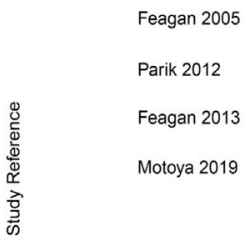

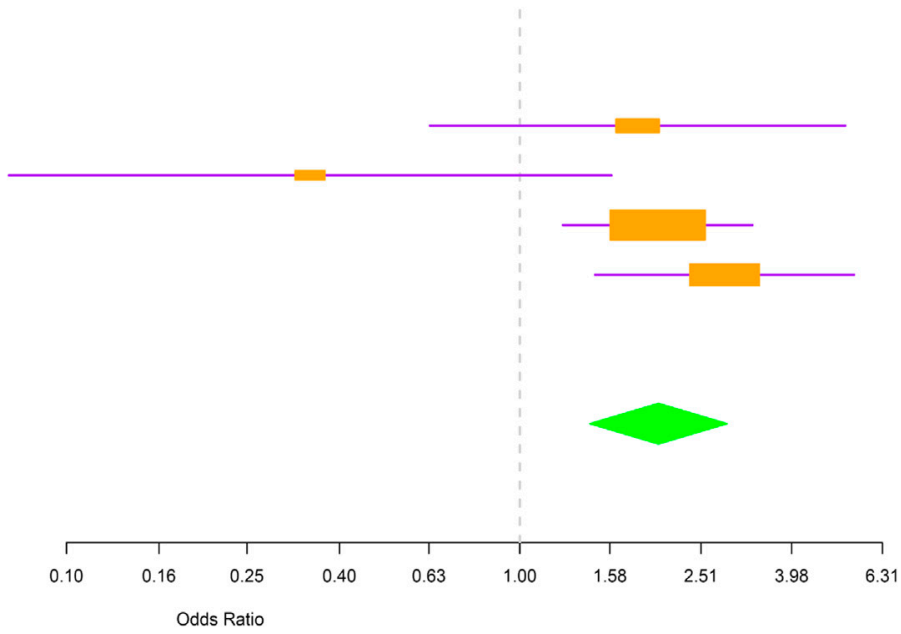

FIGURE 4 | (A) Forest plot for the rate of respiratory tract infections in ulcerative colitis patients. (B) Forest plot for the rate of upper respiratory tract infections in ulcerative colitis patients.

events (e.g., URTI) occurring during treatments and generally lack a placebo group, which makes difficult to ascertain the real risk of RTI.

In conclusion, our study confirms the good safety profile of vedolizumab, even though RTI were more frequent in patients receiving vedolizumab and the risk of URTIs was significantly higher in patients with UC. These findings help clinicians to ensure patients about the safety of vedolizumab especially during the ongoing COVID-19 pandemic, even though a case-by-case decision about continuing/

\section{REFERENCES}

Amiot, A., Serrero, M., Peyrin-Biroulet, L., Filippi, J., Pariente, B., Rablin, X., et al. (2017). One-year effectiveness and safety of vedolizumab therapy for inflammatory bowel disease: a prospective multicentre cohort study. Aliment. Pharmacol. Ther 46, 310-321. doi:10.1111/apt.14167

Ananthakrishnan, A. N., and McGinley, E. L. (2013). Infection-related hospitalizations are associated with increased mortality in patients with withdrawing major disease modifying treatments is always advised.

\section{AUTHOR CONTRIBUTIONS}

IM and ET performed the research, collected and analyzed the data, designed the research study and wrote the paper; IR analyzed the data; GM designed the research study and wrote the paper. All authors approved the final.

inflammatory bowel diseases. J Crohns Colitis 7, 107-112. doi:10.1016/j. crohns.2012.02.015

Bezzio, C., Saibeni, S., Variola, A., Allocca, M., Massari, A., Gerardi, V., et al. (2020). Outcomes of COVID-19 in 79 patients with IBD in Italy: an IG-IBD study. Gut 69 (7), 1213-1217. doi:10.1136/gutjnl-2020-321411

Chaparro, M., Garre, A., Ricart, E., Iborra, M., Mesonero, F., Vera, I., et al. (2018). Short and long-term effectiveness and safety of vedolizumab in inflammatory bowel disease: results from the ENEIDA registry. Aliment Pharmacol. Therapeut 48, 839-851. doi:10.1111/apt.14930 
Christensen, B., Colman, R. J., Micic, D., Gibson, P. R., Goeppinger, S. R., Weber, C. R., et al. (2018). Vedolizumab as induction and maintenance for inflammatory bowel disease: 12-month effectiveness and safety. Inflamm. Bowel Dis 24, 849-860. doi:10.1093/ibd/izx067

Colombel, J. F., Sands, B. E., Rutgeerts, P., Sandborn, W., Danese, S., D'Haens, G., et al. (2017). The safety of vedolizumab for ulcerative colitis and Crohn's disease. Gut 66, 839-851. doi:10.1136/gutjnl-2015311079

Dulai, P. S., Singh, S., Jiang, X., Peerani, F., Narula, N., Chaudrey, K., et al. (2016). The real-world effectiveness and safety of vedolizumab for moderate-severe Crohn's disease: results from the US VICTORY consortium. Am. J. Gastroenterol 111, 1147-1155. doi:10.1038/ajg.2016.236

Downey, C. (2016). Serious infection during etanercept, infliximab and adalimumab therapy for rheumatoid arthritis: a literature review. Int J Rheum Dis 19, 536-550. doi:10.1111/1756-185X.12659

Favale, A., Onali, S., Caprioli, F., Pugliese, D., Armuzzi, A., Macaluso, F. S., et al. (2019). Comparative efficacy of vedolizumab and adalimumab in ulcerative colitis patients previously treated with infliximab. Inflamm. Bowel Dis 25, 1805-1812. doi:10.1093/ibd/izz057

Fantini, B. G., Greenberg, G. R., Wild, G., Fedorak, R. N., Paré, P., McDonald, J. W. D., et al. (2005). Treatment of ulcerative colitis with a humanized antibody to the alpha4beta7 integrin. N. Engl. J. Med 352, 2499-2507. doi:10.1056/ NEJMoa042982

Feagan, B. G., Greenberg, G. R., Wild, G., Fedorak, R. N., Paré, P., McDonald, J. W. D., et al. (2008). Treatment of active Crohn's disease with MLN0002, a humanized antibody to the alpha4beta7 integrin. Clin. Gastroenterol. Hepatol 6, 1370-1377. doi:10.1016/j.cgh.2008.06.007

Feagan, B. G., Rutgeerts, P., Sands, B. E., Hanauer, S., Colombel, J. F., Sandborn, W. J., et al. (2013). Vedolizumab as induction and maintenance therapy for ulcerative colitis. N. Engl. J. Med 369, 699-710. doi:10.1056/ NEJMoa 1215734

Feagan, B. G., Bhayat, F., Khalid, M., Blake, A., and Travis, S. P. L. (2018). Respiratory tract infections in patients with inflammatory bowel disease: safety analyses from vedolizumab clinical trials. J. Crohn's Colitis 12, 905-919. doi:10. 1093/ecco-jcc/jjy047

Godman, B., Haque, M., McKimm, J., Abu Bakar, M., Sneddon, J., Wale, J., et al. (2020). Ongoing strategies to improve the management of upper respiratory tract infections and reduce inappropriate antibiotic use particularly among lower and middle-income countries: findings and implications for the future. Curr. Med. Res. Opin 36, 301-327. doi:10. 1080/03007995.2019.1700947

Guan, W. J., Ni, Z. Y., Hu, Y., Liang, W. H., Ou, C. Q., He, J.-W., et al. (2020). Clinical characteristics of coronavirus disease 2019 in China. N. Engl. J. Med 382, 1708-1720. doi:10.1056/NEJMoa2002032

Hupe, M., Riviere, P., Nancey, S., Roblin, X., Altwegg, R., Filippi, J., et al. (2020). Comparative efficacy and safety of vedolizumab and infliximab in ulcerative colitis after failure of a first subcutaneous anti-TNF agent: a multicentre cohort study. Aliment Pharmacol. Therapeut 51, 852-860. doi:10.1111/apt. 15680

Kennedy, N. A., Jones, G. R., Lamb, C. A., Appleby, R., Arnott, I., Beattie, R. M., et al. (2020). British Society of Gastroenterology guidance for management of inflammatory bowel disease during the COVID-19 pandemic. Gut 69, 984-990. doi:10.1136/gutjnl-2020-321244

Lenti, M. V., Levison, S., Eliadou, E., Willert, R., Kemp, K., Carter, A., et al. (2018). A real-world, long-term experience on effectiveness and safety of vedolizumab in adult patients with inflammatory bowel disease: the cross pennine study. Dig. Liver Dis 50, 1299-1304. doi:10.1016/j.dld.2018. 07.007

Lichtenstein, G. R., Feagan, B. G., Cohen, R. D., Salzberg, B. A., Safdi, M., Popp, J. W., et al. (2018). Infliximab for Crohn's disease: more than 13 Years of real-world experience. Inflamm. Bowel Dis 24, 490-501. doi:10.1093/ibd/ izx072

Long, M. D., Martin, C., Sandler, R. S., and Kappelman, M. D. (2013). Increased risk of pneumonia among patients with inflammatory bowel disease. Am. J. Gastroenterol 108, 240-248. doi:10.1038/ajg.2012.406

Marafini, I., Sedda, S., Pallone, F., and Monteleone, G. (2014). Targeting integrins and adhesion molecules to combat inflammatory bowel disease. Inflamm. Bowel Dis 20, 1885-1889. doi:10.1097/MIB. 0000000000000091

Meserve, J., and Dulai, P. (2020). Predicting response to vedolizumab in inflammatory bowel disease. Front. Med 7, 76. 10.3389/fmed.2020.00076

Monteleone, G., and Ardizzone, S. (2020). Are patients with inflammatory bowel disease at increased risk for Covid-19 infection? J. Crohn's Colitis 14 (9), 1334-1336. doi:10.1093/ecco-jcc/jjaa061

Motoya, S., Watanabe, K., Ogata, H., Kanai, T., Matsui, T., Suzuki, Y., et al. (2019). Vedolizumab in Japanese patients with ulcerative colitis: a Phase 3, randomized, double-blind, placebo-controlled study. PloS One 14, e0212989. doi:10.1371/ journal.pone.0212989

Munster, V. J., Koopmans, M., van Doremalen, N., van Riel, D., and de Wit, E. (2020). A novel coronavirus emerging in China - key questions for impact assessment. N. Engl. J. Med 382, 692-694. doi:10.1056/NEJMp2000929

Parikh, A., Leach, T., Wyant, T., Scholz, C., Sankoh, S., Mould, D. R., et al. (2012). Vedolizumab for the treatment of active ulcerative colitis: a randomized controlled phase 2 dose-ranging study. Inflamm. Bowel Dis 18, 1470-1479. doi:10.1002/ibd.21896

Plevris, N., Chuah, C. S., Allen, R. M., Arnott, I. D., Brennan, P. N., Chaudhary, S., et al. (2019). Real-world effectiveness and safety of vedolizumab for the treatment of inflammatory bowel disease: the scottish vedolizumab cohort. J. Crohn's Colitis 13, 1111-1120. doi:10. 1093/ecco-jcc/jjz042

Prados, A., Muñoz-Fernández, R., Fernandez-Rubio, P., and Olivares, E. G. (2018). Characterization of mesenchymal stem/stromal cells with lymphoid tissue organizer cell potential in tonsils from children. Eur. J. Immunol 48, 829-843. doi:10.1002/eji.201746963

Sandborn, W. J., Feagan, B. G., Rutgeerts, P., Hanauer, S., Colombel, J. F., Sands, M. E., et al. (2013). Vedolizumab as induction and maintenance therapy for Crohn's disease. N. Engl. J. Med 369, 711-721. doi:10.1056/ NEJMoa1215739

Sands, B. E., Feagan, B. G., Rutgeerts, P., Colombel, J. F., Sandborn, W. J., et al. (2014). Effects of vedolizumab induction therapy for patients with Crohn's disease in whom tumor necrosis factor antagonist treatment failed. Gastroenterology 147, 618-e3. doi:10.1053/j.gastro. 2014.05.008

Sarzi-Puttini, P., Marotto, D., Antivalle, M., Salaffi, F., Atzeni, F., Maconi, G., et al. (2020). How to handle patients with autoimmune rheumatic and inflammatory bowel diseases in the COVID-19 era: an expert opinion. Autoimmun. Rev, 102574. doi:10.1016/j.autrev.2020.102574

Scarozza, P., Marafini, I., Laudisi, F., Troncone, E., Schmitt, H., Lenti, M. M., et al. (2020). Extent of mucosal inflammation in ulcerative colitis influences the clinical remission induced by vedolizumab. J. Clin. Med 9, 385. doi:10.3390/ jcm9020385

Shamseer, L., Moher, D., Clarke, M., Ghersi, D., Liberati, A., Petticrew, W., et al. (2015). Preferred reporting items for systematic review and meta-analysis protocols (PRISMA-P) 2015: elaboration and explanation. Bmj 350, g7647. doi:10.1136/bmj.g7647

Solar, D., Chapman, T., Yang, L. L., Wyant, T., Egan, R., and Fedyk, E. R. (2009). The binding specificity and selective antagonism of vedolizumab, an antialpha4beta7 integrin therapeutic antibody in development for inflammatory bowel diseases. J. Pharmacol. Exp. Therapeut 330, 864-875. doi:10.1124/jpet. 109.153973

Syed, M. N., Shah, M., Shin, D. B., Wan, M. T., Winthrop, K. L., and Gelfand, J. M. (2020a). Effect of anti-tumor necrosis factor therapy on the risk of respiratory tract infections and related symptoms in patients with psoriasis-A metaestimate of pivotal phase 3 trials relevant to decision making during the COVID-19 pandemic. J. Am. Acad. Dermatol 84 (1), 161-163. doi:10.1016/j. jaad.2020.08.095

Syed, M. N., Shin, D. B., Wan, M. T., Winthrop, K. L., and Gelfand, J. M. (2020b). The risk of respiratory tract infections in patients with psoriasis treated with interleukin 23 pathway-inhibiting biologics: a meta-estimate of pivotal trials relevant to decision making during the COVID-19 pandemic. J. Am. Acad. Dermatol 83, 1523-1526. doi:10.1016/j.jaad. 2020.06.1014

Watanabe, K., Motoya, S., Ogata, H., Kanai, T., Matsui, T., Suzuki, Y., et al. (2020). Effects of vedolizumab in Japanese patients with Crohn's disease: a 
prospective, multicenter, randomized, placebo-controlled Phase 3 trial with exploratory analyses. J. Gastroenterol 55, 291-306. doi:10.1007/s00535-01901647-w

Yousaf, A., Gayam, S., Feldman, S., Zinn, Z., and Kolodney, M. (2020). Clinical outcomes of COVID-19 in patients taking tumor necrosis factor inhibitors or methotrexate: a multicenter research network study. J. Am. Acad. Dermatol 84 (1), 70-75. doi:10.1016/j.jaad.2020. 09.009

Zhu, N., Zhang, D., Wang, W., Li, X., Yang, B., Song, J., et al. (2020). A novel coronavirus from patients with pneumonia in China, 2019. N. Engl. J. Med 382, 727-733. doi:10.1056/NEJMoa2001017

Zundler, S., Becker, E., Schulze, L. L., and Neurath, M. F. (2019). Immune cell trafficking and retention in inflammatory bowel disease: mechanistic insights and therapeutic advances. Gut 68, 1688-1700. doi:10.1136/gutjnl-2018317977

Conflict of Interest: The authors declare that the research was conducted in the absence of any commercial or financial relationships that could be construed as a potential conflict of interest.

Copyright $\odot 2021$ Marafini, Troncone, Rocchetti and Monteleone. This is an openaccess article distributed under the terms of the Creative Commons Attribution License (CC BY). The use, distribution or reproduction in other forums is permitted, provided the original author(s) and the copyright owner(s) are credited and that the original publication in this journal is cited, in accordance with accepted academic practice. No use, distribution or reproduction is permitted which does not comply with these terms. 